\title{
Academic discussion
}

\section{Comment on the correction of gas-phase signals during IR operando analyses}

\author{
Frederic Meunier* \\ Institut de Recherches sur la Catalyse et l'Environnement de Lyon 2, Avenue Albert Einstein, Villeurbanne Cedex 69626, France
}

In a recent contribution [1] to this journal, Guo and co-workers reported on the design of a dual beam FT-IR spectrometer system aiming, among others, at totally removing the contribution of gas-phase species during operando studies of heterogeneous catalytic reactions. The system consists of two identical cells located in two separate FT-IR spectrometers, one being loaded with a catalyst wafer and the other one being empty and used as a reference signal. The exhaust gas of the sample cell is sent to the reference cell, both being heated up at the same temperature. Any indication on cell volume or the nature of the flow regime (e.g., plug flow or continuously stirred tank reactor (CSTR)) is not mentioned.

This system is flawed for most practical operation conditions when the flow regime is not perfectly that of a CSTR and the system is operated at nonzero conversion levels. This is because the composition of the reactants and products present in the reference cell, which will be that leaving the sample cell, will simply be different from the average composition existing in the sample cell, which will be of intermediate values between that entering and leaving the sampling cell.

Only when reactant conversion is nil, or inert gases are used, as shown in Fig. 3 (in Ref. 1), the gaseous signals of the sample cell and reference cell will exactly cancel out each other.
In contrast, non-zero level of conversion will lead to imperfect gas-phase signal correction. This can be seen in Fig. 7 (in Ref. 1), where the residual rotovibrational spectrum of gas-phase isobutene can be seen in the range $3090-3072 \mathrm{~cm}^{-1}$. Note that this feature was incorrectly assigned by the authors to adsorbates. The minor differences in wavenumbers or band shape observed as compared to the signal of gas-phase isobutene reported in Fig. 4 (in Ref. 1) was due a $150{ }^{\circ} \mathrm{C}$-difference of temperature of sampling, as rotovibrational spectra are temperature-dependent $[2,3]$.

The idea was yet worth investigating and will work, but only if a perfect CSTR regime is obtained for the sampling cell, leading to identical gas composition in the sampling and reference cell.

\section{References}

[1] J. Liu, J. Wang, W. Zhou, C. Miao, G. Xiong, Q. Xin, H. Guo, Chin. J. Catal., 2017, 38, 13-19.

[2] A. Paredes-Nunez, I. Jbir, D. Bianchi, F. C. Meunier, Appl. Catal. A, 2015, 495, 17-22.

[3] P. Atkins, J. de Paula, Atkin's Physical Chemistry, 8th ed., Oxford University Press, Oxford, 2006, 457.

\section{Responses to the comments of Prof. Frederic Meunier}

\author{
Jiaxu Liu a, Qin Xin b, Hongchen Guo a, \\ ${ }^{a}$ Department of Catalytic Chemistry and Engineering \& State Key Laboratory of Fine Chemicals, Dalian University of Technology, Dalian 116012, \\ Liaoning, China \\ ${ }^{b}$ State Key Laboratory of Catalysis, Dalian Institute of Chemical Physics, Chinese Academy of Sciences, Dalian 116023, Liaoning, China
}

\footnotetext{
* Corresponding author. Tel: +33-472445468; E-mail: fcm@ircelyon.univ-lyon1.fr Received 8 September 2018; Accepted 24 October 2018; Published 5 January 2019. DOI: 10.1016/S1872-2067(18)63188-2 | http://www.sciencedirect.com/science/journal/18722067 | Chin. J. Catal., Vol. 40, No. 1, January 2019
} 
Dear Prof. Frederic Meunier,

Thanks for your straightforward comments and constructive suggestions on our recent study on dual-beam FT-IR spectroscopy published in Chin. J. Catal., 2017, 38, 13-19. We are glad to reply your questions and to give some supplementary remarks concerning the dual beam FT-IR spectrometer, its usage and application progress.

The first question you posed is about the nature of the feed gas flow regime in sample cell (e.g., plug flow or CSTR-like). You worry about a non-CSTR flow regime in the sample cell of our dual beam IR-cell reactor, which will result in difference in the gas composition of sample cell and reference cell, and consequently an imperfect gas-phase correction.

Your second question is related to the IR absorption bands in Fig. 7 in the range of $3092-3080 \mathrm{~cm}^{-1}$. You believe that we made a mistake by assigning the bands in between 3092-3080 $\mathrm{cm}^{-1}$ to adsorbates (stretching of the $\mathrm{C}-\mathrm{H}$ bond of benzene ring), which, in your opinion, should be rotovibrational spectra of gas-phase isobutene molecules (3099-3072 cm-1, see Fig. 4), and the bands difference between the range of 3092-3080 $\mathrm{cm}^{-1}$ in Fig. 7 and 3099-3072 cm-1 in Fig. 4 was simply a temperature effect.

As you said, the inequivalence of the gas-phase compositions in both sample cell and reference cell is a big obstacle of perfect gas-phase correction for dual beam FT-IR spectroscopy study. We have encountered this problem at the very beginning of building the spectroscopic system. The problem was solved during the design of dual beam IR-cell reactor by trial and er-

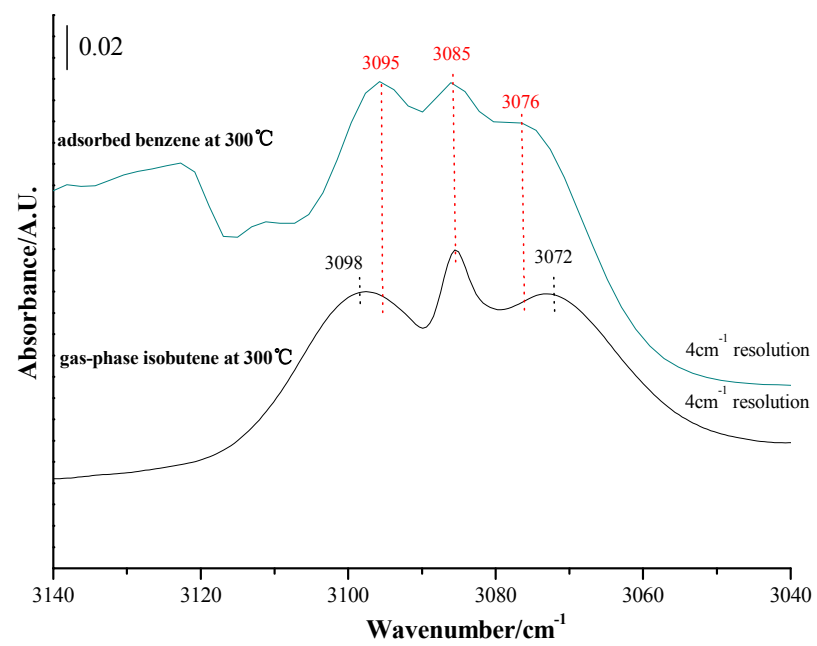

Fig. R1. Spectrum of benzene adsorption under the conditions of $300{ }^{\circ} \mathrm{C}$ $0.1 \mathrm{MPa}$ on nano-sized HZSM-5 recorded at time-on-stream (TOS) $=15$ minutes of benzene introduction $(50 \mathrm{ml} / \mathrm{min}$ nitrogen carrier gas bubbling through a benzene tank at $25^{\circ} \mathrm{C}$ before entering dual beam IR-cell reactor), and spectrum of gas-phase isobutene ( $6 \%$ isobutene in nitrogen, $50 \mathrm{ml} / \mathrm{min}$ ) recorded at $300{ }^{\circ} \mathrm{C}$. All the spectra were obtained with the dual beam FT-IR spectrometer. Among them, the spectrum of adsorbed benzene was obtained by the spectrum subtraction of sample cell and reference cell, while the spectrum of gas-phase isobutene was obtained using empty sample cell. ror. In short, the gas flow in the sample cell (also the reference cell) of the IR-cell reactor, with a Reynolds number of as large as $2.73 \times 10^{6}$, is in turbulent region. Moreover, the turbulent gas is forced to turn-round in the sample cell before leaving for reference cell. So we think the flow regime in our IR-cell reactor is similar to CSTR.

In order to reply your second question, we carried out some comparative experiments, and the results are shown in Fig. R1. We think our former attribution on the $3092-3080 \mathrm{~cm}^{-1}$ IR bands in Fig. 7 is supported by the obtained spectra. As can be seen in these spectra, the adsorption of benzene on H-ZSM-5 zeolite does produce absorptions in this region, and the three bands, viz., 3076, 3085, $3095 \mathrm{~cm}^{-1}$ agree well with the bands in the same region in Fig. 7. Literature [1] has taught that the stretching of $\mathrm{C}-\mathrm{H}$ bond of adsorbed benzene ring would produce these bands. It is true that gas-phase isobutene also gives similar absorptions in this region, but the three bands of isobutene gas molecule are 3072, 3085 and $3098 \mathrm{~cm}^{-1}$, respectively. Please pay attention to the bands at 3072 and $3098 \mathrm{~cm}^{-1}$. They shift toward different directions too much compared with the corresponding bands of benzene adsorption on catalyst surface. So, the band group of adsorbed benzene at 3076, 3085, $3095 \mathrm{~cm}^{-1}$ is different from that of gas-phase isobutene molecule.

In order to know the effect of temperature on the gas-phase molecular spectrum, we also conducted an auxiliary experiment which is exhibited in Fig. R2. These spectra confirm that the increase of temperature cannot transform the 3072, 3085 and $3098 \mathrm{~cm}^{-1}$ bands of isobutene to 3076, 3085 and 3095 $\mathrm{cm}^{-1}$. The rotovibrational spectra of the $\mathrm{C}-\mathrm{H}$ bond of gas-phase isobutene molecules could be seen at the resolution of $0.5 \mathrm{~cm}^{-1}$. However, in the spectra shown in Fig. 7, the rotovibrational spectra were absence as the $\mathrm{C}-\mathrm{H}$ bond of the surface adsorbed isobutene molecular could not rotate. In this regard, we are willing to have further discussion with you and glad to receive your reference.

The prototype of our dual beam FT-IR spectrometer used in

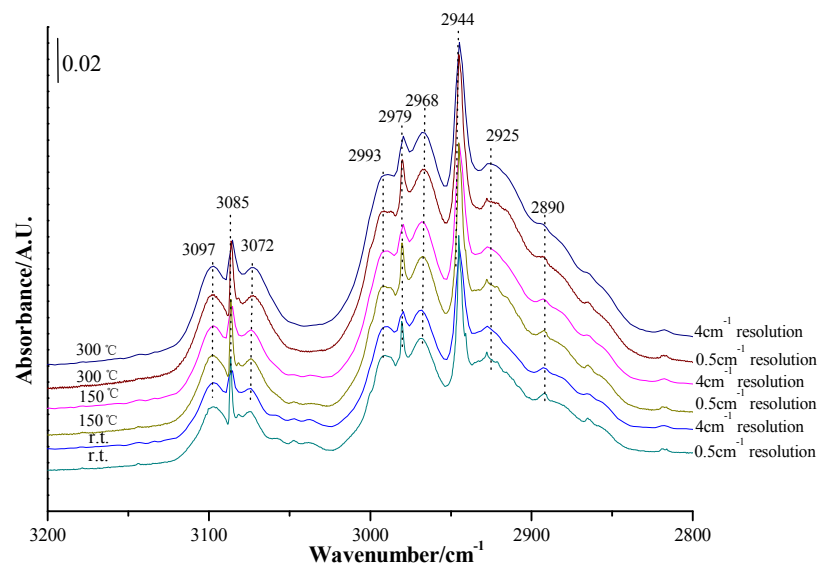

Fig. R2. Spectra of gas-phase isobutene (6\% isobutene in nitrogen, 50 $\mathrm{ml} / \mathrm{min}$ ) recorded at different temperature with different resolutions using the empty sample cell of the dual beam FT-IR spectrometer. 


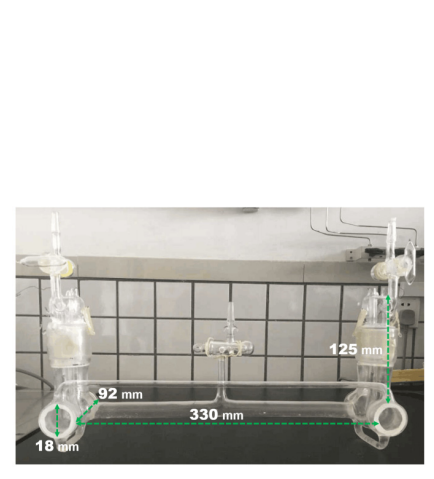

(A)

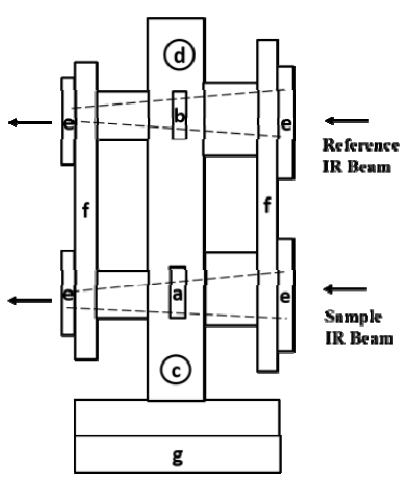

(B)
Fig. R3. Image of the quartz dual beam IR cell prototype (A) used in the study of Chin. J. Catal., 2017, 38, 13-19, and the dual beam IR cell schematic (B) given by A. T. Bell [2-4]: (a) sample disk; (b) reference disk; (c) gas inlet; (d) gas outlet; (e) KBr windows; (f) cooling channels; (g) cover flange.

the previous study was built on the basis of two identical interferometer IR optical systems, which, for the sake of risk control, were still kept in separated state. Consequently, the matched dual beam IR cell is relatively long. From Fig. R3 we can see that the distance of the two cells was $330 \mathrm{~mm}$. This means that the gas-phase of reference cell will lag behind that of sample cell by a short time interval which must be taken under consideration in spectrum subtraction. But it was already the shortest distance the two separated optical systems could approach each other. To our joy, however, the crude dual beam IR cell, worked in the same way as Bell's [2-4], collaborated so well with the simple spectrometer prototype that it allowed us to have finished our exploratory work plan.

In addition to the isobutene conversion reported previously, most recently we have also obtained interesting results with aromatization of propylene, isobutane and propane over Zn/H-ZSM-5 [5] and ZnSnPt/H-ZSM-5, epoxidation of propylene with $\mathrm{H}_{2} \mathrm{O}_{2}$ over titanosilicate molecular sieve as well as characterizations of weak adsorptions on surface of solid catalysts. All of these gave us confidence to go further.

Our future plan is to integrate the two identical interferometer IR optical systems, and meanwhile to design new dual beam IR cell with extremely small dead volume for fast response and pressure hull for pressurized reactions.

\section{References}

[1] Sivasankar, S. Vasudevan, J. Phys. Chem. B, 2004, 108, 1158511590.

[2] E. L. Force. A. T. Bell, J. Catal., 1975, 38, 440-460.

[3] E. L. Force, A. T. Bell, J. Catal., 1975, 40, 356-371.

[4] E. L. Force, A. T. Bell, J. Catal., 1976, 44, 175-182.

[5] J. Liu, N. He, W. Zhou, L. Lin, G. Liu, C. Liu, J. Wang, Q. Xin, G. Xiong, H. Guo, Catal. Sci. Technol., 2018, 8, 4018-4029.

\section{Graphical Abstract}

Chin. J. Catal., 2019, 40: 1-3 doi: 10.1016/S1872-2067(18)63188-2

Comment on the correction of gas-phase signals during IR operando analyses

Frederic Meunier*

Institut de Recherches sur la Catalyse et l'Environnement, France

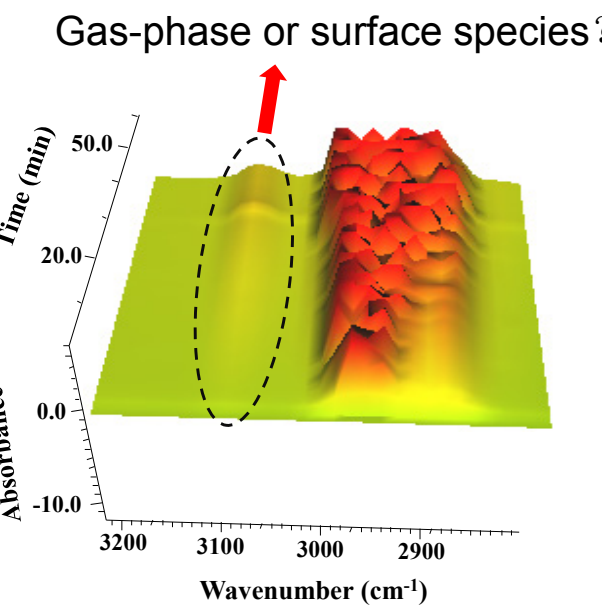

The accuracy of dual beam Fourier transform infrared (DB-FTIR) spectrometer in eliminating the interference of gas-phase molecular vibration in gas/solid heterogeneous catalysis under reaction conditions is discussed. 